Sara Naghib

Cynthia van der Starre

Saskia J. Gischler

Koen F. M. Joosten

Dick Tibboel

\section{Mortality in very long-stay pediatric intensive care unit patients and incidence of withdrawal of treatment}

Received: 3 April 2009

Accepted: 17 September 2009

Published online: 24 October 2009

(C) Copyright jointly hold by Springer and ESICM 2009

S. Naghib - C. van der Starre .

S. J. Gischler · K. F. M. Joosten .

D. Tibboel $(\bullet)$

Department of Intensive Care, Erasmus

Medical Centre, Sophia Children's Hospital,

P.O. Box 2060, 3000 CB Rotterdam,

The Netherlands

e-mail: d.tibboel@erasmusmc.nl

Tel.: +31-10-7036567

Fax: +31-10-7036288

S. Naghib

e-mail: saranaghib@hotmail.com

C. van der Starre

Department of Neonatology, Erasmus

Medical Centre, Sophia Children's Hospital,

Rotterdam, The Netherlands

\begin{abstract}
Background: The mortality for children with prolonged stay in pediatric intensive care units (PICU) is much higher than overall mortality. The incidence of withdrawal or limitation of therapy in this group is unknown. Purpose: To assess mortality and characteristics of children admitted for $\geq 28$ days to our ICU, and to describe the extent to which limitations of care were involved in the terminal phase preceding death. Methods: For the period 2003 to 2005 clinical data were collected retrospectively for children with prolonged stay (defined as $\geq 28$ days) in a medical/surgical PICU of a university children's hospital. Results: In the PICU, $4.4 \%$ of the children $(116 / 2,607$, equal gender, mean age 29 days) had a prolonged
\end{abstract} occupied $63 \%$ of total admission days. Mortality during admission for this group was five times higher $(22 \%)$ than the average PICU mortality rate of $4.6 \%$. Withdrawal or limitation of therapy preceded $70 \%$ of deaths. Conclusions: Children with prolonged stay in the PICU have a significantly high risk of mortality. Death is typically preceded by limitation of care.

Keywords Long-stay patient . Pediatric intensive care unit . End of life - Mortality ·

Limitations of care

\section{Abbreviations \\ LSP Long-stay patient \\ PICU Pediatric intensive care unit}

\section{Introduction}

Critical appraisal of the effectiveness of different modes of treatment and demands for more cost-efficient hospital processes have focused attention on the duration of care in an intensive care unit (ICU), an environment which necessarily provides high levels of care and therefore requires substantial operating budgets [1-3]. Median stay for most patients is 2 days [4,5], but a small minority need to stay much longer and use resources in excess of their numeric proportions [6]. Long stay in the pediatric
ICU is usually defined as stay longer than 12-13 days [1, $3,7]$. A special subgroup is formed by patients with very prolonged stay, longer than 30 days $[7,8]$.

The few reports available on outcomes of long-stay pediatric ICU patients demonstrate higher mortality and morbidity compared with short-stay patients $[3,6,9,10]$. Withdrawal and limitation of medical care is associated with $14-75 \%$ of deaths in neonatal and pediatric intensive care [11-13]. These issues have been extensively discussed in the past decade $[8,11,12,14-16]$ and have been the subject of ongoing public discourse. The extent to 
which limitations of care actually contribute to death in very long-stay pediatric ICU patients is not known. The aim of the present study was to assess characteristics and mortality of very long-stay patients in our unit and to describe how often treatment was limited and/or withdrawn.

\section{Methods}

Data collection and definitions

The ICU of the Erasmus MC-Sophia Children's Hospital, Rotterdam, is a level III interdisciplinary intensive care unit for children in The Netherlands, providing all pediatric and surgical subspecialties (except direct cardiopulmonary bypass). All patients, including newborns with major congenital anomalies, admitted from 1 January 2003 until 31 December 2005 were retrospectively identified using the computerized patient data management system. Long-stay patients were defined as those admitted for at least 28 continuous days. The reason for this cutoff point was that 28 days is three times the median length of stay in our unit. Whenever a long-stay patient was readmitted, only the first admission was included in the study. The following clinical data were collected from hospital medical records and our patient data management system: age, sex, presence and number of congenital malformations, reason for admission, and diagnosis. Both data systems are used by nursing and medical staff.

Primary outcome was death during admission. Deaths during operations or other procedures were classified as intensive care deaths. Cause of death was categorized as [13]: brain death, do-not-resuscitate, failed cardiopulmonary resuscitation, withdrawal or limitation of therapy [17-20].

Retrospectively, every patient's death was classified according to four categories:

1. Brain death $(\mathrm{BD})$ : when criteria for brain death were fulfilled.

2. Do-not-resuscitate (DNR): when a previously ordered DNR document was available.

3. Failed resuscitation (RES): when advanced life support failed.

4. Withdrawal or limitation of therapy (W/LT): when, by agreement between family and medical staff, present level of life-sustaining treatment (LST) was limited and/or inotropes/mechanical ventilation removed.

Main diagnoses were categorized into six groups: disorders of the respiratory system, gastrointestinal disorders, multiple congenital abnormalities, neurological disorders, cardiac disease, and others. Data on survival, limitations of therapy, and withdrawal of therapy were collected. Decisions regarding do-not-resuscitate (DNR) and limitation/withdrawal of life-sustaining therapy (W/LT) were taken by a multidisciplinary team. As described earlier $[21,22]$ families were involved in all cases.

Severity of illness on admission was measured by PRISM III score according to Pollack et al. published in 1996, which was calculated from physiological data that included the most abnormal values in the first $12 \mathrm{~h}$ and second $12 \mathrm{~h}$ of PICU stay.

In the terminal phase, patients were not transferred to a different ward. Instead, optimal palliative care was offered in a separate part of the intensive care unit. When needed or requested by the medical team and/or parents, the institutional Ethics Review Board was consulted. Approval from this board for the present study was waived due to its retrospective character.

\section{Setting}

The setting for this study was a 34-bed multidisciplinary tertiary level III pediatric ICU with extra corporeal membrane oxygenation (ECMO) facilities, including a 6-bed step-down unit. This ICU is part of a 250-bed pediatric university hospital with a referral population of 4,000,000 and staffed full-time by intensive care specialists with basic training in pediatrics or anesthesiology.

\section{Data analysis}

Data are presented as mean \pm standard deviation (SD) or median (interquartile range [IQR] or range) where appropriate and were analyzed by using SPSS software (SPSS version 12.0 for Windows, 2005, Chicago, IL).

\section{Results}

During the study period 2,607 patients were admitted on 3,700 occasions for a total of 16,013 admission days. Of this group $4.4 \%(116 / 2,607)$ patients were identified as very long-stay patients, responsible for $3.4 \%(126 / 3,700)$ of total admissions and consuming 63\% (10,055/16,013) of admission days. One hundred and six long-stay patients were admitted once, seven were admitted twice, and three were admitted three times.

Demographic and care characteristics of long-stay patients are summarized in Table 1. Clinical diagnoses were: disorders of the respiratory system $(29,25 \%)$, gastrointestinal disorders $(23,20 \%)$, multiple congenital abnormalities $(19,16 \%)$, neurological disorders (18, $15 \%)$, cardiac disease $(18,15 \%)$, and others $(9,8 \%)$. 
Table 1 Demographic characteristics of long-stay patients

\begin{tabular}{lccc}
\hline Demographic characteristics & $\begin{array}{l}\text { Long-stay patients } \\
N=116\end{array}$ & $\begin{array}{l}\text { Long-stay nonsurvivors } \\
N=25\end{array}$ & $\begin{array}{l}\text { Long-stay survivors } \\
N=91\end{array}$ \\
\hline Admissions & 126 & 25 & 111 \\
Fraction of total admissions (\%) & 3 & 2.6 & 0.6 \\
Males (\%) & 57 & 60 & 1 \\
Age median (months) (IQR 25) & 1 & 29 & 1 \\
Mean age (months) & 29 & - & 29 \\
Mortality (\%) & 22 & 36 & - \\
Surgical patients (\%) & 37 & 33 & 45 \\
Median ventilation (days) (IQR 25) & 30 & & 45 \\
\hline
\end{tabular}

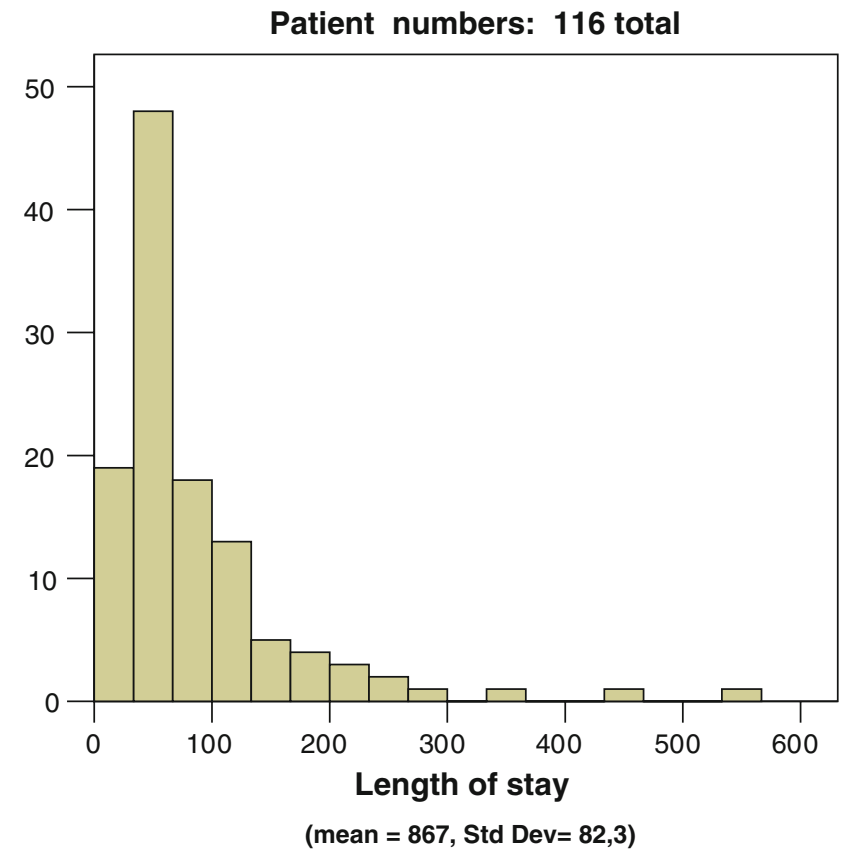

Fig. 1 Length of intensive care unit stay in days for a total of 116 long-stay patients

Their median length of stay was 56 (IQR 37-108) days. Distribution of length of stay is shown in Fig. 1. Outliers were three patients admitted for more than 300 days, the longest stay being 546 days. PRISM III scores are shown in Fig. 2. The majority of the long-stay patients $(58 \%)$ had a PRISM III score between 0 and 5 . Ninety-four patients $(81 \%)$ had a maximum PRISM III score of 10 .

During the study period $4.6 \%(120 / 2,607)$ of the total patient group died during admission; 21\% (25/120) of them were very long-stay patients. The mortality rate of long-stay patients was higher compared with short-stay patients $\left[\begin{array}{lllll}22 \% & (25 / 116) & \text { versus } & 3.8 \% & (95 / 2,491) \text {, }\end{array}\right.$ $p<0.001]$. The characteristics of long-stay survivors and nonsurvivors are shown in Table 2. Neonates accounted for half of the patient population in both groups. The most common diagnoses among the long-stay nonsurvivors

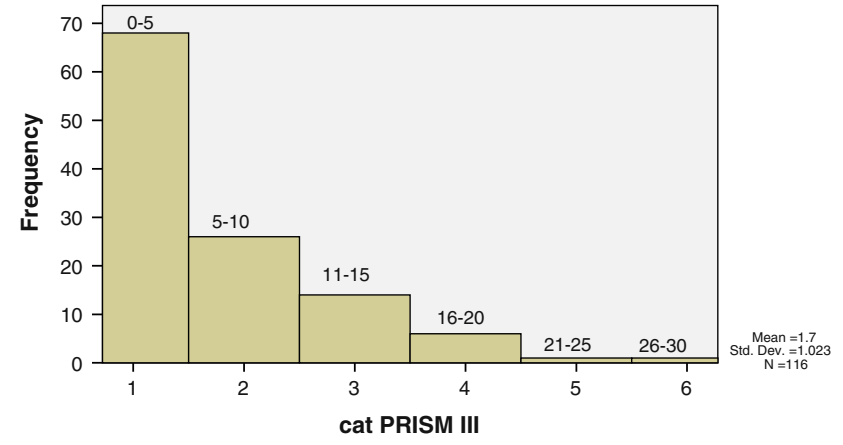

Fig. 2 PRISM III score among long-stay patients

Table 2 Characteristics of nonsurvivors and survivors

\begin{tabular}{lll}
\hline & Nonsurvivors & Survivors \\
\hline Patient numbers & 25 & 91 \\
Patients no./total admissions & 25 & $91 / 101$ \\
Male (\%) & 60 & 58 \\
Median age (days) & 29 & 34 \\
Median length of stay (days) & 67 & 54 \\
Patient admission days & 2,538 & 7,517 \\
Neonate (\%) & 52 & 47 \\
Ex-premature (\%) & 8 & 5 \\
Diagnosis (\%) & $2 / 25(8 \%)$ & $22 / 91(24 \%)$ \\
Resp. & $7 / 25(28 \%)$ & $7 / 91(8 \%)$ \\
Cardio. & $4 / 25(16 \%)$ & $17 / 91(18 \%)$ \\
Gastro. & $4 / 25(16 \%)$ & $14 / 91(15 \%)$ \\
Neuro. & $1 / 25(4 \%)$ & $6 / 91(7 \%)$ \\
Others. & $7 / 25(28 \%)$ & $25 / 91(27 \%)$ \\
MCA $^{\mathrm{a}}$ & & \\
\hline
\end{tabular}

${ }^{a}$ Multiple congenital anomalies

were multiple congenital anomalies $(7 / 25,28 \%)$ and cardiovascular disease $(7 / 25,28 \%)$. Multiple congenital anomalies was the most frequent diagnosis among the long-stay survivors as well $(25 / 91,27 \%)$, followed by diseases of the respiratory system $(22 / 91,24 \%)$.

The specific primary diagnoses which led to long stay are presented in Table 3. A total of 101 readmissions were counted for 91 survivors. Comparing different 
Table 3 Specific primary diagnosis among long-stay patients

\begin{tabular}{lcc}
\hline Primary diagnosis & Nonsurvivors & Survivors \\
\hline Dilated/restricted cardiomyopathy & 2 & 1 \\
Congenital heart disease & 7 & 6 \\
Acquired arrhythmia & 0 & 1 \\
Congenital airway/pulmo. disease & 1 & 4 \\
Acquired airway/pulmo. disease & 0 & 5 \\
Congenital gastrointestinal & 3 & 12 \\
$\quad$ malformations & 0 & \\
Acquired gastrointestinal disease & 1 & 5 \\
Congenital hypotonia & 1 & 1 \\
Status epilepticus (therapy resist) & 0 & 1 \\
Acquired neurological disease & 0 & 1 \\
Central hypoventilation & 1 & 3 \\
Infantile encephalopathy & 1 & 1 \\
Psychomotor retardation eci & 1 & 2 \\
Metabolic disease & 0 & 1 \\
Trauma & 0 & 1 \\
Sepsis & 0 & 4 \\
Malignancy & 7 & 25 \\
Multiple congenital anomalies & 0 & 12 \\
Congenital diaphragmatic hernia & 0 & 1 \\
Hematological/vascular disease & 25 & 91 \\
Total &
\end{tabular}

diagnosis between survivors and nonsurvivors showed no statistical significance $(p=0.999)$.

Figure 3 shows a flow diagram in which end-of-life categories are shown for nonsurvivors. No patients were included in the category of brain death. Among 25 deaths, 6 patients had DNR status. Active withdrawal of support occurred in 12 patients: 2 with DNR orders and 10 without. Three patients were removed from extracorporeal life support (ECLS): one patient after 12 days ECMO and two cardiac patients with refractory shock. Table 4 summarizes characteristics in terms of mode-of-death category and end-of-life treatment.

All end-of life meetings between the medical team and family which resulted in limitations of treatment, DNR or withdrawal were documented. The cause of death was well documented in all the charts, including an electronic patient data management system which has been used by both nursing and medical staff members. Consensus between caregivers and medical team regarding the endof-life meetings was reached in all cases. All parents were physically present with their child when passing away, except one parent couple who, with respect to their religious restrictions, unfortunately could not be present at the time of death.

\section{Discussion}

In this study the group of very long-stay patients (LOS $\geq 28$ days) forms only a small proportion of the total

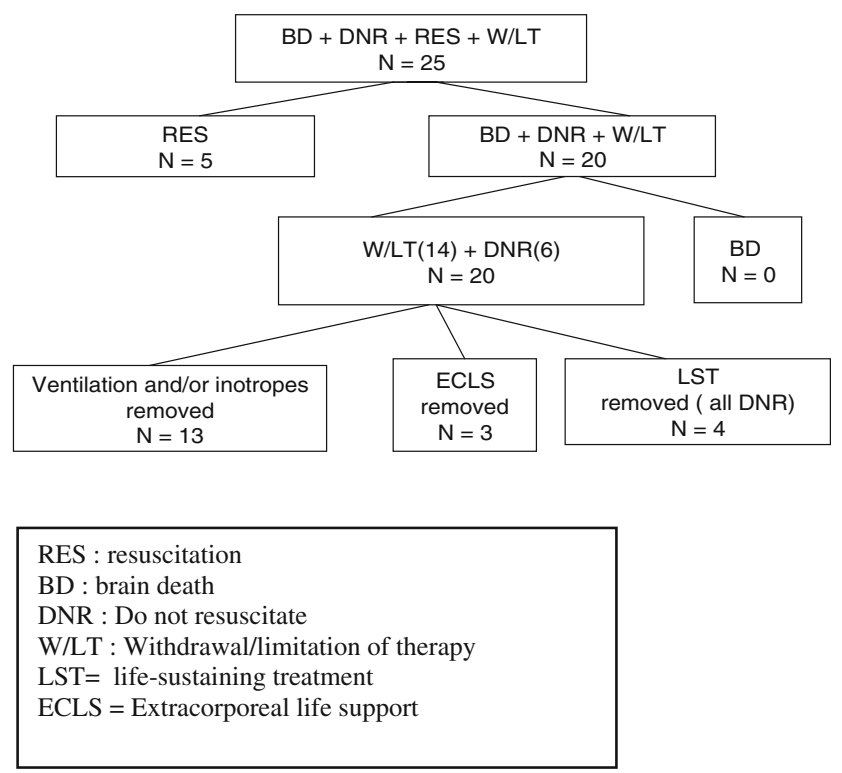

Fig. 3 Nonsurvivors end-of-life flow diagram in different patient groups, in which all nonsurvivors are categorized according to mode of death. RES: resuscitation, BD: brain death, DNR: do not resuscitate, W/LT: withdrawal/limitation of therapy, LST: lifesustaining treatment; ECLS: extracorporeal life support. In addition the W/LT and DNR group $(n=20)$ is categorized by different ICU dependent treatments which they had been receiving

cohort (3\%), but they are responsible for a considerable part of admission days (63\%). The mortality rate for this group was five times higher than that for the total cohort.

We found that our very long-stay patients consumed a high proportion of total admission days, which is consistent with earlier studies performed in adult and pediatric ICUs (LOS $>12$ days) $[3,7,20]$. The overall mortality rate in our ICU is comparable to the mortality rates reported by European and North American studies $[1,4,13]$. We reported a much higher mortality rate in very long-stay patients (defined as $>28$ days) compared with short-stay patients. It is difficult to compare this mortality with earlier reports, as these were studies of long-stay patients (defined as 7-30 days) [3, 6, 9, 10]. However, it is still lower than the ICU mortality documented among adults following very long stay (32\%) [7].

Previous analysis by Marcin et al. [1] of diverse PICUs in the USA indicates that, among other factors, PRISM III score between 10 and 33 was predictive of long stay in their population. Given that PRISM III score has not been evaluated amongst European long-stay patients, we considered these scores in our study sample. The majority $(81 \%)$ of our long-stay patients did have a PRISM III score between 0 and 10 , which is at the lower range of the score and might be explained by the unique case mix of our ICU and the PRISM III score not being population independent. 
Table 4 Characteristics in mode-of-death categories

\begin{tabular}{|c|c|c|c|c|}
\hline & $\mathrm{BD}$ & DNR & W/LT & RES \\
\hline Number & 0 & 6 & 14 & 5 \\
\hline Age (years) mean (SD) & & $2.3(4.9)$ & $2.5(5.6)$ & $2.4(5.3)$ \\
\hline Age (years) median (range) & & $0.0(0.0-3.9)$ & $0.0(0.0-1.5)$ & $0.0(0.0-6.0)$ \\
\hline LOS (days) mean (SD) & & $82(72)$ & $102(134)$ & $123(51)$ \\
\hline LOS (days) median (range) & & $48(39-136)$ & $62(33-107)$ & $131(75-167)$ \\
\hline \multicolumn{5}{|c|}{ Initial ICU admission diagnosis $(n)$} \\
\hline Resp & & 3 & 7 & - \\
\hline Cardiac & & - & 4 & - \\
\hline Resp + Cardiac & & - & 1 & - \\
\hline Resp + Surgery & & 2 & 2 & - \\
\hline Neurology & & 1 & - & - \\
\hline \multicolumn{5}{|c|}{ End-of-life treatments and characteristics } \\
\hline Ventilated patients $(n)$ & & 6 & 14 & - \\
\hline Ventilation days $80-100 \%$ & & 4 & 12 & - \\
\hline Tracheostomy & & 2 & 4 & - \\
\hline Noninvasive ventilation & & - & - & \\
\hline Multiple congenital anomaly & & 4 & 5 & - \\
\hline Dialysis & & - & - & - \\
\hline
\end{tabular}

$D N R$ do not resuscitate

$R E S$ (failed) resuscitation

The few studies available of adults who required at least 28-30 days of ICU care generally report reasonable to relatively good chance of hospital and long-term survival, with some disability during daily activities [7, 2327]. Friedrich et al. [7] reported a $32 \%$ ICU mortality and a $58 \%$ hospital survival rate among their very long-stay patients. Most survivors were discharged to their previous place of residence, which was considered as an important indicator of quality of life.

A high proportion of deaths in this study were preceded by end-of-life discussions, resulting in withdrawal or limitation of life-sustaining treatment. So far, however, there are no guidelines or protocols to facilitate the decision-making process when establishing appropriate boundaries concerning the extent of medical care. Once certainty about the diagnosis and prognosis has been obtained, it is vital that a prominent member of the interdisciplinary teams informs the parents and evaluates whether the treatment given is in the child's best interest $[12,15,22,28,29]$. If disagreement occurs between the parents and the view held by the medical team, this conflict can be mediated according to the guidelines of the Dutch Pediatric Society, reported in the early 1990s and available to all its members. Fortunately disagreement did not occur in our patient group.

Optimal palliative care was provided in a separate part of the intensive care unit without transferring the patient to a different ward. In the holistic approach towards our patients we appreciate continuous care given by the same care providers. Caring for families with a child awaiting the end of life creates a situation where an inevitable death demands the involved care providers to continue a
$W / L T$ withdrawal/limitation of therapy

$B D$ brain death close relationship, especially when a long stay has been involved.

The retrospective character of the study is a limitation. It was conducted in a mixed ICU population, which limits its generalizability and application to other centers. Moreover, our unit's infrastructure and the lack of separate high-dependency units within our hospital may have had a decisive influence on our findings. The issue of generalizability, given the different population and institutional setting, has also been raised by Friedrich et al. in 2006 when evaluating an adult ICU population [7]. They described the unique character of their data, which may not be applicable to other centers with a different view on health care organization [7]. Having an intermediate/stepdown unit and long-term ventilatory facilities apart from the intensive care might yield other results (for example, shorter ICU length of stay). The 28-day minimum length of stay we defined may limit the applicability of our findings to other patient populations with severe congenital malformations with shorter length of stay but also high mortality.

Notwithstanding the limitations of this type of investigation, we believe that our data on very long-stay patients will raise awareness of this matter and contribute towards the improvement and establishment of appropriate goals of care. Long-term survival, functional outcomes, and quality of life are important aspects of PICU patients that need more study. Pediatric follow-up data in long-stay patients are limited and contradictory [3, $6]$.

In conclusion, the high mortality rate and frequent application of a "withholding" approach shown in this 
study emphasizes the necessity of timely care assessment, when a patient's stay in the pediatric ICU exceeds 28 days. A multidisciplinary team should then discuss possibilities of cure and care based on current and predicted future suffering. We recommend the use of a transparent individualized protocol to guide the treatment team towards boundaries of care.

Ongoing investigation is needed to point out the different indications and justifications of limitations of treatment for pediatric ICU patients with prolonged length of stay. Early identification of patients at risk of very long stay and recognition of their high risk of mortality and potential consequences for future therapeutic modalities should be incorporated into the activities of teams working at the pediatric ICU.

Conflict of interest statement None.

\section{References}

1. Marcin JP, Slonim AD, Pollack MM, Ruttimann UE (2001) Long-stay patients in the pediatric intensive care unit. Crit Care Med 29:652-657

2. Slonim AD, Marcin JP, Pollack MM (2003) Long-stay patients: are there any long-term solutions? Crit Care Med 31:313-314

3. Pollack MM, Wilkinson JD, Glass NL (1987) Long-stay pediatric intensive care unit patients: outcome and resource utilization. Pediatrics 80:855-860

4. Gemke RJ, Bonsel GJ (1995) Comparative assessment of pediatric intensive care: a national multicenter study. Pediatric Intensive Care Assessment of Outcome (PICASSO) Study Group. Crit Care Med 23:238245

5. Ruttimann UE, Pollack MM (1996) Variability in duration of stay in pediatric intensive care units: a multiinstitutional study. J Pediatr 128:35-44

6. van der Heide P, Hassing MB, Gemke RJ (2004) Characteristics and outcome of long-stay patients in a paediatric intensive care unit: a case-control study. Acta Paediatr 93:1070-1074

7. Friedrich JO, Wilson G, Chant C (2006) Long-term outcomes and clinical predictors of hospital mortality in very long stay intensive care unit patients: a cohort study. Crit Care 10:R59

8. Tibballs J (2007) Legal basis for ethical withholding and withdrawing lifesustaining medical treatment from infants and children. J Paediatr Child Health 43:230-236

9. Kanter RK, Bove EL, Tobin JR, Zimmerman JJ (1986) Prolonged mechanical ventilation of infants after open heart surgery. Crit Care Med 14:211-214

10. Gemke RJ, Bonsel GJ, van Vught AJ (1995) Long-term survival and state of health after paediatric intensive care. Arch Dis Child 73:196-201
11. Levetown M, Pollack MM, Cuerdon TT, Ruttimann UE, Glover JJ (1994) Limitations and withdrawals of medical intervention in pediatric critical care. JAMA 272:1271-1275

12. Hazebroek FW, Tibboel D, Mourik M, Bos AP, Molenaar JC (1993) Withholding and withdrawal of life support from surgical neonates with life-threatening congenital anomalies. J Pediatr Surg 28:1093-1097

13. Garros D, Rosychuk RJ, Cox PN (2003) Circumstances surrounding end of life in a pediatric intensive care unit. Pediatrics 112:e371

14. Gill M (2005) PICU prometheus: ethical issues in the treatment of very sick children in pediatric intensive care. Mortality 10:262-275

15. Verhagen AA, Sauer PJ (2005) End-oflife decisions in newborns: an approach from The Netherlands. Pediatrics 116:736-739

16. Mink RB, Pollack MM (1992)

Resuscitation and withdrawal of therapy in pediatric intensive care. Pediatrics 89:961-963

17. Parker BL, Frewen TC, Levin SD, Ramsay DA, Young GB, Reid RH, Singh NC, Gillett JM (1995) Declaring pediatric brain death: current practice in a Canadian pediatric critical care unit. CMAJ 153:909-916

18. Guidelines for the appropriate use of do-not-resuscitate orders (1991) Council on Ethical and Judicial Affairs, American Medical Association. JAMA 265:1868-1871

19. Guidelines for the diagnosis of brain death. Patient Care Policy (1987) Toronto, Ontario, Canada; Hospital for Sick Children (Report No. 2.25)

20. Do not resuscitate (DNR) order (1994) In: Policy and Procedure Manual Hospital for Sick Children. Toronto, Ontario, Canada. 1-3
21. Hazebroek FW, Bouman NH, Tibboel D (2001) The neonate with major malformations: experiences in a university children's hospital in the Netherlands. Semin Pediatr Surg 10:222-229

22. Caniano DA, Hazebroek FW, DenBesten KE, Tibboel D (1995) Endof-life decisions for surgical neonates: experience in The Netherlands and United States. J Pediatr Surg 30:14201424

23. Rimachi R, Vincent JL, Brimioulle S (2007) Survival and quality of life after prolonged intensive care unit stay. Anaesth Intensive Care 35:62-67

24. Gracey DR, Naessens JM, Krishan I, Marsh HM (1992) Hospital and posthospital survival in patients mechanically ventilated for more than 29 days. Chest 101:211-214

25. Montuclard L, Garrouste-Orgeas M, Timsit JF, Misset B, De Jonghe B, Carlet J (2000) Outcome, functional autonomy, and quality of life of elderly patients with a long-term intensive care unit stay. Crit Care Med 28:3389-3395

26. O'Brien BP, Butt W, Suhr H, Bimpeh Y, McKenna AM, Bailey MJ, Scheinkestel CD (2006) The functional outcome of patients requiring over 28 days of intensive care: a long-term follow-up study. Crit Care Resusc 8:200-204

27. Hughes M, MacKirdy FN, Norrie J, Grant IS (2001) Outcome of long-stay intensive care patients. Intensive Care Med 27:779-782

28. Verhagen E, Sauer PJ (2005) The Groningen protocol-euthanasia in severely ill newborns. N Engl J Med 352:959-962

29. Bondi SA, Gries D, Faucette K (2006) Neonatal euthanasia? Pediatrics 117:983-984 